\title{
Editorial for the JECR special issue on all solid-state batteries
}

\author{
Jennifer L. M. Rupp ${ }^{1} \cdot$ Daniel Rettenwander $^{2} \cdot$ John $_{\text {Kilner }}{ }^{3} \cdot$ Marca Doeff $^{4}$
}

Published online: 24 August 2017

(C) Springer Science+Business Media, LLC 2017

The solid-state batteries considered here operate with ceramic electrolyte materials such as oxides, sulfides or phosphates and offer a new paradigm for batteries with high specific energy and power density. While ceramic electrolytes eliminate potentially flammable liquids and corrosive environments, which has profoundly positive implications for improved safety, many challenges remain. To fully realize the promise of high energy density, new cell and electrode designs are needed. Furthermore, strategies for mitigating dendrite formation as well as accelerating electrode-electrolyte interface kinetics is essential. Suitable electrodes tolerating high chemo-mechanical stresses induced during charging and discharging also must be identified, and an improved understanding of the defect chemistry operative in the bulk and at interfaces is required. Successful design and operation of such temperature-robust ceramic cells have far-reaching implications for new applications like synergetic operations taking advantage of industrial waste heat in large-scale battery storage units, electric vehicles, and grid storage.

At the other extreme of scale, there are solid-state microbatteries, which can compete with today's

Jennifer L. M. Rupp

jrupp@mit.edu

1 Department of Materials Science and Engineering, Massachusetts Institute of Technology, Cambridge, MA 02139, USA

2 Institute for Chemical Technology, Graz University of Technology, Graz, Austria

3 Department of Materials, Imperial College London, London SW7 2AZ, UK

4 Energy Storage and Distributed Resources Division, Lawrence Berkeley National Laboratory, Berkeley, CA 94720, USA supercapacitors in electronics, and power sensors. To achieve an improved understanding of transfer kinetics, the study of solid-solid electrode-electrolyte interfaces requires fundamental attention. Novel "in-operando" characterization techniques are essential for obtaining insights into electro-chemo-mechanical phenomena associated with lithiation/delithiation processes and their impact on phase and mechanical stability associated with volumetric changes. No matter what form the devices take, the discovery and development of better ceramic electrolytes, both with respect to ionic conductivity and stability over wide chemical activity limits are high priorities. Turning from the macro to the nano, the study of grain vs. grain boundary contributions, as well as interfacial reactions driven by, e.g., interactions with humidity and $\mathrm{CO}_{2}$, and the impact on chemical, and structural stability, as well as electrochemical performance, is critical for the understanding and optimization of solid-state batteries. Improving the understanding of the fundamentals of surface segregation, and its impact on surface chemistry, are also critical in the development of novel microbatteries as well as large scale systems. Finally, a sound understanding of the processing of ceramic electrolytes and electrodes to mass produce thin mechanically robust layers is a fundamental requirement for commercialization. The use of electroceramic components in particular, creates opportunities for consideration of novel high storage capacity electrode materials, which may not be stable in liquid or corrosive media such as those found in standard liquid-based batteries, but whose reactivity is not an issue in the solid-state.

In this special issue of the Journal of Electroceramics, we present a selection of invited and contributed articles, which focus on all solid-state battery materials and device aspects. These papers span a wide range of themes, 
including novel solid state electrolyte Li-ion conductors, understanding the solid state electrochemical interface, evolution of lithium morphologies during battery performance or ceramic processing strategies that are potentially inexpensive and allow mass production of large scale cells or microbatteries. We are fortunate to have attracted 12 high quality and timely contributions to this issue.

An invited review on the relevance and a realistic view of solid electrolytes for lithium-based batteries by Lotsch and Maier leads off the special issue [1]. In this paper, the pros and cons for replacing classic liquid electrolytes by solid electrolytes are critically discussed by the authors. A general overview on $\mathrm{Li}$-ion conducting electroceramic materials and recent advances in the field of superionic conductors, i.e., LGPS (lithium germanium phosphosulfide) and other materials is provided, with focus on their conduction mechanisms, structures, phases and mechanical stability. Following this work, Uitz, Epp, Bottke and Wilkening discuss $\mathrm{Li}$-ion dynamics in solid electrolytes for Li-ion batteries, probed by time-domain nuclear magnetic resonance (NMR), together with conductivity measurements [2]. The information on activation energies and jump rate vs. local structures provides insights on Li-ion diffusion pathways for garnets, phosphates and sulfides.

Braun, Uhlmann, Weber, Störmer, Gerthsen and IversTiffee discuss the separation of bulk and grain boundary contributions to the total conductivity of $\mathrm{Li}_{3 \mathrm{x}} \mathrm{La}_{2 / 3-\mathrm{x}} \mathrm{TiO}_{3}$ solidstate electrolytes [3]. Cheng, Hou, Lux, Kostecki, Davis, Zorba, Mehta, and Doeff offer new insights into the role of sintering atmosphere and its impact on the structural, chemical and electrical properties of lithium-oxide garnet-based ceramics [4]. The authors report on new sintering engineering strategies designed to alter the Li-ion conductivity by changing grain boundary chemistry and oxygen vacancy concentration; valuable for large-scale battery synthesis of garnet-based electrolytes. Wachter-Wetzl, Wagner, Rettenwander, Taibl, Amthauer and Fleig then introduce microelectrodes for locally probing electrical conductivity and the effect of moisture and chemical inhomogenities for lithium-oxide garnets [5].

Van den Broek, Rupp, and Afyon focus on the solid state electrode-electrolyte interface with respect to Li-ion transfer, storage capacity, and chemo-mechanical stability for large-scale ceramic processing, and report on low cost processing routes designed to realize lithium titanate oxide anodes coupled with lithium-oxide garnet electrolytes with high discharge capacities [6]. Kubanska, Castro, Tortet, Dollé, and Bouchet investigate ceramic lithium half battery cells with differing thicknesses of the electrode using NASICON-type electrolytes [7]. Following this work, Ulhenbruck, Dornseiffer, Lobe, Dellen, Tsai, Gotzen, Sebold, Finsterbusch, and Guillon investigate the phase stability of $\mathrm{LiCoO}_{2}$ cathodes with Li-garnet electrolytes showing that physical vapor deposition or liquid precursor processing and fast thermal processing are promising processing routes towards achieving stable electrode-electrolyte structures [8]. $\mathrm{Wu}, \mathrm{El} \mathrm{Kazzi}$, and Villevieille reveal the reactivity of $\mathrm{Li}_{3} \mathrm{PS}_{4}$ electrolytes with various anodes such as lithium titanates, lithium metal, and indium-lithium alloys through post-mortem analysis [9]. The electroplastic properties of $\mathrm{LiMn}_{2} \mathrm{O}_{4}$ spinels are examined in the work of McGrogan, Chiang, and Van Vliet [10]. These authors demonstrated that by the partial substitution of the transition metal ionic dopant on the manganese lattice site, the mechanical properties such as hardness and elastic modulus can be significantly altered in the spinel to design-in fracture toughness of solid state batteries.

Turning to thin film batteries and microbattery structures, Dudney explores the evolution of lithium morphology during cycling of thin film solid-state batteries with Lipon electrolytes [11]. Significant changes between wrinkled and pitted surfaces are reported for lithium anodes on Lipon electrolytes, which may occur for thin film batteries during cycling, presumably due to lithium dewetting and stress changes. Finally, Chen, Eichel, and Notten review metalorganic chemical vapor deposition processing for all solidstate lithium microbatteries [12].

For our concluding remarks, the guest editors highlight the fact that solid-state batteries and their electroceramic components allow not only for excellent performance but also fascinating new discoveries of phases, structures, chemo-mechanical phenomena, and conduction mechanisms. Ultimately, these are the key to safer battery energy storage and define the future. We hope to motivate material scientists and engineers to go to the next level, designing new all solid-state cathode-electrolyte interfaces based on sustainable and abundant materials. To date, most solid-state batteries rely on socio-economically and -politically critical elements in the cathodes. As exemplified in this special issue, the science and engineering of solid-state batteries have reached a remarkably sophisticated level. The next frontier is sustainability; let's accept this as a new challenge!

Acknowledgements All authors and contributors are thanked by the guest editors for their high quality work, critical discussion and the sharing of scientific concepts and discoveries through this special issue. Special thanks go to Prof. Harry Tuller, not only for giving us the opportunity to publish this exciting special issue on solid-state batteries in the Journal of Electroceramics, but also for his dedication and continued contributions to the Electroceramics field.

\section{References}

1. B.V. Lotsch, J. Maier, Relevance of solid electrolytes for lithiumbased batteries: a realistic view. J. Electroceram. (2017). doi:10. 1007/s10832-017-0091-0

2. M. Uitz, V. Epp, P. Bottke, M. Wilkening, Ion dynamics in solid electrolytes for lithium batteries Probing jump rates and activation energies through time-domain Li NMR. J. Electroceram. (2017). doi:10.1007/s10832-017-0071-4 
3. P. Braun, C. Uhlmann, A. Weber, H. Störmer, D. Gerthsen, E. IversTiffée, Separation of the bulk and grain boundary contributions to the total conductivity of solid lithium-ion conducting electrolytes. J. Electroceram. (2017). doi:10.1007/s10832-016-0061-y

4. L. Cheng, H. Hou, S. Lux, R. Kostecki, R. Davis, V. Zorba, A. Mehta, M. Doeff, Enhanced lithium ion transport in garnet-type solid state electrolytes. J. Electroceram. (2017). doi:10.1007/ s10832-017-0080-3

5. A. Wachter-Welzl, R. Wagner, D. Rettenwander, S. Taibl, G. Amthauer, J. Fleig, Microelectrodes for Local Conductivity and Degradation Measurements on al Stabilized Li7La3Zr2O12 Garnets. J. Electroceram. (2016). doi:10.1007/s10832-016-0058-6

6. J.V.D. Broek, J.L.M. Rupp, S. Afyon, Boosting the Electrochemical Performance of li-Garnet Based All-Solid-State Batteries with Li4Ti5O12 Electrode: Routes to Cheap and Large Scale Ceramic Processing. J. Electroceram. (2017). doi:10.1007/s10832-0170079-9

7. A. Kubanska, L. Castro, L. Tortet, M. Dollé, R. Bouchet, Effect of composite electrode thickness on the electrochemical performances of all-solid-state li-ion batteries. J. Electroceram. (2017). doi:10. 1007/s10832-017-0088-8
8. S. Uhlenbruck, J. Dornseiffer, S. Lobe, C. Dellen, C.L. Tsai, B. Gotzen, D. Sebold, M. Finsterbusch, O. Guillon, Cathodeelectrolyte material interactions during manufacturing of inorganic solid-state lithium batteries. J. Electroceram. (2016). doi:10.1007/ s10832-016-0062-X

9. X. Wu, M. El Kazzi, C. Villevieille, Surface and Morphological Investigationof the Electrode/Electrolyte Properties in an AllSolid-State Battery Using a Li2S-P2S5 Solid Electrolyte. J. Electroceram. (2017). doi:10.1007/s10832-017-0084-Z

10. F.P. McGrogan, Y.M. Chiang, K.J. Van Vliet, Effect of Transition Metal Substitution on Elastoplastic Properties of LiMn2O4 Spinel. J. Electroceram. (2016). doi:10.1007/s10832-016-0057-7

11. N.J. Dudney, Evolution of the Lithium Morphology from Cycling of Thin Film Solid State Batteries. J. Electroceram. (2017). doi:10. 1007/s10832-017-0073-2

12. C. Chen, R.A. Eichel, P.H.L. Notten, Metal-Organic Chemical Vapor Deposition Enabling All-Solid-State li-ion Microbatteries: A Short Review. J. Electroceram. (2017). doi:10.1007/s10832017-0090-1 